\title{
Injectivity onto a star-shaped set for local homeomorphisms in $n$-space
}

\author{
by Gianluca Gorni (Udine) and Gaetano Zampieri (Padova)
}

\begin{abstract}
We provide a number of either necessary and sufficient or only sufficient conditions on a local homeomorphism defined on an open, connected subset of the $n$-space to be actually a homeomorphism onto a star-shaped set. The unifying idea is the existence of "auxiliary" scalar functions that enjoy special behaviours along the paths that result from lifting the half-lines that radiate from a point in the codomain space. In our main result this special behaviour is monotonicity, and the auxiliary function can be seen as a Lyapunov function for a suitable dynamical system having the lifted paths as trajectories.
\end{abstract}

1. Introduction. Calculus textbooks teach that a one-variable differentiable function whose derivative never vanishes over an interval cannot take twice the same value, or at least this wisdom is implicit in Rolle's theorem. As one steps into more advanced calculus, the Jacobian matrix replaces the derivative in its linear approximation meaning, but the fact must be faced that the invertibility of the Jacobian matrix guarantees injectivity only locally, with the complex exponential readily given as a counterexample.

It is an interesting and important problem to find useful conditions on the triple domain/codomain/local homeomorphism that ensure that we are dealing with a bijection, or, at least, an injection. Quite some work has been devoted to the case when the topological spaces involved are open subsets of Banach or finite-dimensional Euclidean spaces. A sample of papers on the subject is contained in the reference list at the end of this paper, and

1991 Mathematics Subject Classification: Primary 26B10.

Key words and phrases: global invertibility, local homeomorphisms, star-shaped sets, line-lifting, Lyapunov functions.

Gianluca Gorni did most of his part in this work while visiting the Department of Mathematics of Chūō University, Tōkyō, supported by the "Ministero della Università e della Ricerca Scientifica e Tecnologica" (MURST) and by the "European Communities Scientific Training Programme in Japan" and is greatly indebted to Prof. Hitoshi Ishii and to all of Chūō University for their hospitality.

Gaetano Zampieri was supported during this research project by the "Consiglio Nazionale delle Ricerche" (CNR). 
we are not going to comment specifically on many of them. What almost all of them share is that they explore the local homeomorphism by drawing a continuous path in the codomain space and then seeing what happens if we try to patch up a continuous path in the domain space by repeatedly applying local inverses of the mapping, a procedure called "path-lifting". We call "local inverses" of a local homeomorphism the inverses of restrictions of the mapping to an open subset where the mapping is one-to-one.

In the approach that we follow here we restrict the attention to the lifting of segments of straight lines ("line-lifting") that radiate from a single given point of the codomain space. Then we try to derive some a priori estimate on the lifted paths by postulating the existence of a suitable scalar "auxiliary" function on the domain space. In this way we are able to provide either necessary and sufficient or only sufficient conditions for a local homeomorphism or diffeomorphism to be injective and for the image to be a star-shaped set. The most general of the results, which makes minimal restrictions on the shape of domain and image, can be stated as Theorem 1.1 below.

THEOREM 1.1. Let $\Omega$ be a nonempty, open and connected subset of $\mathbb{R}^{n}$, $x_{0}$ be a point of $\Omega, f: \Omega \rightarrow \mathbb{R}^{n}$ be a local homeomorphism and $y_{0}:=f\left(x_{0}\right)$. Then the following two conditions are equivalent:

(a) $f$ is one-to-one and $f(\Omega)$ is star-shaped with respect to $y_{0}$;

(b) there exists a function $k: \Omega \rightarrow \mathbb{R}$ which is continuous, proper and bounded from below and such that, for any local inverse $g$ of $f$ and any vector $v \in \mathbb{R}^{n}$, the real function

$$
t \mapsto k\left(g\left(y_{0}+t v\right)\right)
$$

is locally weakly increasing at all the $t>0$ where it is defined.

The reader who is familiar with dynamical systems will probably notice that the conditions that we impose on the auxiliary function $k$ of this theorem look very much like the definition of a Lyapunov function. Indeed, in Proposition 2.6 we will show that the theorem can be reformulated as a result about global asymptotic stability of the equilibrium for a suitable dynamical system, whose trajectories are the lifted paths and for which $k$ is a Lyapunov function. The auxiliary functions that appear in other invertibility results that we will prove do not lend themselves either readily or at all to a Lyapunov interpretation: namely, Propositions 4.2 and 4.3 on injectivity with star-shaped image and Propositions 2.9 and 4.4 on injectivity with $f(\Omega)=\mathbb{R}^{n}$.

Theorem 1.1 remains true if both the $f$ in the preamble and the $k$ in condition (b) are required to be either locally Lipschitz continuous or to be $m$ times continuously differentiable, $1 \leq m \leq \infty$. This is because, given 
condition (a), we can obtain a suitable $k$ by composing $f$ with a regular scalar function defined on $f(\Omega)$, as will be shown in Section 3 .

Whenever the theorem applies, the composition $k \circ f^{-1}$ is weakly increasing along the half-lines departing from $y_{0}$. Hence, the sets of the family $\{x \in \Omega \mid k(x)<c\}, c>\inf k$, are all open and connected, and they are mapped by $f$ onto sets that are star-shaped with respect to $y_{0}$.

One possible use of the theorem is to verify whether a given local homeomorphism is injective by checking condition (b) against promising auxiliary functions $k$. If both $f^{-1}$ and $k$ are smooth, the monotonicity part of (b) translates, of course, into a pointwise condition involving the first derivatives of $f$ and $k$ :

Proposition 1.2. Let $\Omega$ be a nonempty, open subset of $\mathbb{R}^{n}, f: \Omega \rightarrow \mathbb{R}^{n}$ be a $C^{1}$ local diffeomorphism and $k: \Omega \rightarrow \mathbb{R}$ be a function which is $C^{1}$ on $\Omega \backslash\left\{x_{0}\right\}$. Then the next two conditions are equivalent:

1) the function $t \mapsto k\left(g\left(y_{0}+t v\right)\right)$ is locally weakly increasing at the $t>0$ where it is defined, for any choice of a vector $v \in \mathbb{R}^{n}$ and of a local inverse $g$ of $f$;

2) the following relation holds:

$$
k^{\prime}(x) f^{\prime}(x)^{-1}\left(f(x)-f\left(x_{0}\right)\right) \geq 0 \quad \text { for all } x \in \Omega \backslash\left\{x_{0}\right\} .
$$

Applying Proposition 1.2 with the particular function $k(x):=1 /\left(r_{0}-\right.$ $\left.\left|x-x_{0}\right|\right)$ on the open ball $B_{r_{0}}\left(x_{0}\right):=\left\{x \in \mathbb{R}^{n}|| x-x_{0} \mid<r_{0}\right\}$, we can easily turn the implication (b) $\Rightarrow$ (a) of Theorem 1.1 into the following proposition, which can in particular be used as a sufficient test for injectivity of a smooth function on a ball:

Proposition 1.3. Let $f: B_{r_{0}}\left(x_{0}\right) \rightarrow \mathbb{R}^{n}$ be a $C^{1}$ local diffeomorphism. Then $f$ is one-to-one and $f\left(B_{r}\left(x_{0}\right)\right)$ is star-shaped with respect to $f\left(x_{0}\right)$ for any $r \leq r_{0}$ if and only if

$$
\left(x-x_{0}\right) \cdot f^{\prime}(x)^{-1}\left(f(x)-f\left(x_{0}\right)\right) \geq 0 \quad \text { for all } x \in B_{r_{0}}\left(x_{0}\right) .
$$

A local $C^{1}$ diffeomorphism defined in an arbitrary neighbourhood of $x_{0}$ will be injective with star-shaped image with respect to $f\left(x_{0}\right)$ on any open ball $B_{r}\left(x_{0}\right) \subset \Omega$ where inequality (1.3) holds. Notice that, although the auxiliary function $k$ used in the proof depends on the radius of the ball, formula (1.3) does not. This is because $k \circ f^{-1}$ is weakly increasing along the radii departing from $f\left(x_{0}\right)$ if and only if the same holds for $\left|f^{-1}\right|$.

Proposition 1.3 can also be obtained, along a somewhat different line of thought, as a corollary of a result of [26], which states that if $\left(x-x_{0}\right)$. $f^{\prime}\left(x_{0}\right)^{-1}\left(f(x)-f\left(x_{0}\right)\right) \geq 0$ for all $x$ such that $\left|x-x_{0}\right|=r<r_{0}$, then $f$ is one-to-one on $B_{r}\left(x_{0}\right)$ and $f\left(B_{r}\left(x_{0}\right)\right)$ is star-shaped with respect to $f\left(x_{0}\right)$. 
A weaker version of that result will also be proved in this paper as Proposition 4.2 .

The statement of Proposition 1.3 specializes as follows in the case of holomorphic functions of one complex variable, with the usual identification of $\mathbb{R}^{2}$ with $\mathbb{C}$. (The operator $\mathfrak{R}$ is the real part.)

Proposition 1.4. Let $D_{r_{0}}=\left\{z \in \mathbb{C}|| z \mid<r_{0}\right\}$ be a disk in $\mathbb{C}$ and $f: D_{r_{0}} \rightarrow \mathbb{C}$ be a holomorphic function with $f(0)=0$ and with never vanishing derivative. Then $f$ is one-to-one on $D$ and $f\left(D_{r}\right)$ is star-shaped with respect to $0 \in \mathbb{C}$ for all $r<r_{0}$ if and only if

$$
\mathfrak{R} \frac{\bar{z} f(z)}{f^{\prime}(z)} \geq 0 \quad \text { for all } z \in D .
$$

As a result, this is not particularly novel, since it is a variation of a wellknown theorem on "starlike" holomorphic functions (see [8], Theorem 2.10). What seems notable in Proposition 1.4 is the fact that it is unmistakably a real variables result. This helps making out that in the proof of [8] only the following property is genuinely complex-analytic: if a one-to-one holomorphic function $f: D_{r_{0}} \rightarrow \mathbb{C}$ has nonvanishing derivative and $f\left(D_{r_{0}}\right)$ is star-shaped with respect to $f(0)$, then all the images $f\left(D_{r}\right), r \leq r_{0}$, of the smaller disks are also star-shaped with respect to $f(0)$. Of course $C^{\infty}$ real diffeomorphisms do not share this feature.

A simple consequence of Proposition 1.3 is the following general property of $C^{1}$ local diffeomorphisms.

Proposition 1.5. Let $\Omega$ be a nonempty open subset of $\mathbb{R}^{n}, x_{0}$ be a point of $\Omega$ and $f: \Omega \rightarrow \mathbb{R}^{n}$ be a $C^{1}$ local diffeomorphism. Then there exists $r_{0}>0$ such that $f\left(B_{r}\left(x_{0}\right)\right)$ is star-shaped with respect to $f\left(x_{0}\right)$ for all $r \leq r_{0}$.

Just compute

$$
\begin{aligned}
(x- & \left.x_{0}\right) \cdot f^{\prime}(x)^{-1}\left(f\left(x_{0}\right)-f(x)\right) \\
& =\left(x-x_{0}\right) \cdot\left(f^{\prime}\left(x_{0}\right)^{-1}+o(1)\right)\left(f^{\prime}\left(x_{0}\right)\left(x_{0}-x\right)+o\left(\left|x-x_{0}\right|\right)\right) \\
& =-\left|x-x_{0}\right|^{2}+o\left(\left|x-x_{0}\right|^{2}\right) \quad \text { as } x \rightarrow x_{0}
\end{aligned}
$$

and notice that formula (1.3) is true for $r$ small enough. In the special case of one-variable holomorphic functions a lot is known about just how large the $r_{0}$ of Proposition 1.5 can be ("radius of starlikeness"; see [8]). On the other hand, except for the trivial one-dimensional case, the mere existence of an $r_{0}$ is lost if the $C^{1}$ regularity of $f$ is relaxed to bilipschitz continuity, as we will show in Example 4.1. Incidentally, the kind of mappings described in that example arises independently in connection with the topological equivalence of the flows of linear ordinary differential equations. 
The remainder of the paper is organized as follows. In Section 2, we will start by introducing a machinery of line-lifting, and then proceed to reformulate the problem of injectivity with star-shaped image as a problem of either global liftability or global stability; among the applications we will prove part $(\mathrm{b}) \Rightarrow(\mathrm{a})$ of Theorem 1.1. The converse implication will be shown in Section 3 through a detailed study of the geometry of open, star-shaped sets. Except for the bilipschitz Example 4.1, Section 4 applies the local homeomorphism theory of Section 2 to local diffeomorphisms. In particular, we will obtain some variants of the results on invertibility in the large for local diffeomorphisms that one of the authors had developed in [26] and [27]. The original proofs had used ideas from the stability theory for ordinary differential equations.

2. The theory for a local homeomorphism. Throughout this section $\Omega$ will be a connected open set in $\mathbb{R}^{n}, x_{0}$ a point of $\Omega, f: \Omega \rightarrow \mathbb{R}^{n}$ a local homeomorphism and $y_{0}=f\left(x_{0}\right)$.

Our proof of the implication (b) $\Rightarrow$ (a) of Theorem 1.1 will use the concept of line-lifting, which is the problem of finding continuous paths in $\Omega$ which are mapped into straight lines by $f$. We will start by stating two preliminary propositions about existence, uniqueness, dependence on parameters and asymptotic behaviour. We omit the proofs, which are elementary. Most of the first proposition would actually hold even if we replaced the right-hand side $f(x)+t v$ of equation (2.1) with an arbitrary continuous function of the couple $(x, t v)$.

Proposition 2.1. For every $x \in \Omega$ and $v \in \mathbb{R}^{n}$ there exists one and only one interval $I_{x, v}$ containing $0 \in \mathbb{R}$ and one and only one continuous function $\gamma_{x, v}: I_{x, v} \rightarrow \Omega$ such that

$$
\gamma_{x, v}(0)=x \quad \text { and } \quad f\left(\gamma_{x, v}(t)\right)=f(x)+t v \quad \text { for all } t \in I_{x, v},
$$

and such that if $J$ is another interval containing 0 and $\widetilde{\gamma}: J \rightarrow \Omega$ is a continuous function for which $f(\widetilde{\gamma}(t))=f(x)+$ tv for $t \in J$, then $J \subset I$ and $\widetilde{\gamma}$ is the restriction of $\gamma_{x, v}$ to $J$. Moreover, the set $D:=\{(x, v, t) \mid x \in \Omega$, $\left.v \in \mathbb{R}^{n}, t \in I_{x, v}\right\}$ is open in $\Omega \times \mathbb{R}^{n} \times \mathbb{R}$, the mapping $(x, v, t) \mapsto \gamma_{x, v}(t)$ is continuous on $D$ and for any fixed $x \in \Omega$ and $t \neq 0$ the mapping $v \mapsto \gamma_{x, v}(t)$ is an open mapping on $\left\{v \in \mathbb{R}^{n} \mid(x, v, t) \in D\right\}$. Finally, $\gamma_{x, v}(t)$ depends on $(v, t)$ only through the product $t v$, that is, if $(x, v, t) \in D$ and $t v=t^{\prime} v^{\prime}$ then $\left(x, v^{\prime}, t^{\prime}\right) \in D$ and $\gamma_{x, v}(t)=\gamma_{x, v^{\prime}}\left(t^{\prime}\right)$.

Note that if $f$ is a local $C^{1}$ diffeomorphism, the implicit function problem (2.1) is equivalent to the following ordinary differential equation (Ważewski's equation [25]):

$$
\gamma_{x, v}(0)=x \quad \text { and } \quad \frac{d}{d t} \gamma_{x, v}(t)=f^{\prime}\left(\gamma_{x, v}(t)\right)^{-1} v \quad \text { for all } t \in I_{x, v} .
$$


Next, we exhibit a property that holds because we are lifting lines and not just arbitrary continuous paths.

Proposition 2.2. Let $C$ be a compact subset of $\Omega, x \in \Omega$ and $v \in \mathbb{R}^{n}$, $v \neq 0$. Then there exists $\bar{t} \in I_{x, v}, \bar{t} \geq 0$, such that $\gamma_{x, v}(t) \in \Omega \backslash C$ for all $t \in I_{x, v}, t \geq \bar{t}$.

The title theme "invertibility and star-shaped image" of this paper has an obvious affinity with the "line" half of "line-lifting", because $f(\Omega)$ is starshaped with respect to $y_{0}$ if and only if the straight line segment that joins $y_{0}$ to any $f(x) \in f(\Omega)$ is contained in $f(\Omega)$. A bit less trivial is the "lifting" connection, that can be described as follows: whenever $f(\Omega)$ is star-shaped with respect to $y_{0}$, then $f$ is one-to-one if and only if all those radii shooting from $y_{0}$ toward $f(x)$ can be globally lifted to continuous paths radiating from $x_{0}$. The next lemma couches this fact in a more technical language.

Lemma 2.3. Let $A$ be a connected subset of $\Omega$ containing $x_{0}$. Then the following conditions are equivalent:

(a) $f$ is one-to-one on $A$ and $f(A)$ is star-shaped with respect to $y_{0}$;

(b) $1 \in I_{x_{0}, f(x)-y_{0}}$ and $\gamma_{x_{0}, f(x)-y_{0}}(t) \in A$ for all $x \in A$ and all $t \in[0,1]$;

(c) $1 \in I_{x_{0}, f(x)-y_{0}}, \gamma_{x_{0}, f(x)-y_{0}}(t) \in A$ and $\gamma_{x_{0}, f(x)-y_{0}}(1)=x$ for all $x \in A$ and all $t \in[0,1]$.

Proof. To help understand the formulas, note that the path $\gamma_{x_{0}, f(x)-y_{0}}$ is the lifting, emanating from $x_{0}$, of the radius that departs at time $t=0$ from $y_{0}$ and is scheduled to arrive in $f(x)$ at time $t=1$. Saying that $1 \in$ $I_{x_{0}, f(x)-y_{0}}$ means that the lifting is successful on the whole segment up to and including $f(x)$.

(a) $\Rightarrow(\mathrm{b})$. If (a) holds and $x \in A$, then $y_{0}+t\left(f(x)-y_{0}\right) \in f(A)$ for all $t \in[0,1]$ because $f(A)$ is star-shaped and $\gamma_{x_{0}, f(x)-y_{0}}(t)=\left(\left.f\right|_{A}\right)^{-1}\left(y_{0}+\right.$ $\left.t\left(f(x)-y_{0}\right)\right) \in A$ for all $t \in[0,1]$ because $\left.f\right|_{A}$ is invertible with continuous inverse.

(b) $\Rightarrow$ (c). Consider the set $B:=\left\{x \in A \mid \gamma_{x_{0}, f(x)-y_{0}}(1)=x\right\}$. This set is nonempty because $x_{0} \in B$ for sure. It is closed in $A$ because it is defined by an equality between the two continuous functions $x \mapsto \gamma_{x_{0}, f(x)-y_{0}}(1)$ and $x \mapsto x$, both defined on the whole of $A$. To see that $B$ is also open in $A$, let $\bar{x} \in B$ and let $U$ be an open neighbourhood of $\bar{x}$ in $\Omega$ on which $f$ is one-to-one. The set $V:=\left\{x \in U \cap A \mid \gamma_{x_{0}, f(x)-y_{0}}(1) \in U\right\}$ is contained in $U \cap A$, it is open in $A$ and it contains $\bar{x}$. The mapping $f$ is also one-to-one on $V$, so that for any $x \in V$ we can write

$$
\begin{aligned}
\gamma_{x_{0}, f(x)-y_{0}}(1)=x & \Leftrightarrow f\left(\gamma_{x_{0}, f(x)-y_{0}}(1)\right)=f(x) \\
& \Leftrightarrow y_{0}+1\left(f(x)-y_{0}\right)=f(x) \\
& \Leftrightarrow f(x)=f(x),
\end{aligned}
$$

and conclude that $V \subset B$. 
(c) $\Rightarrow\left(\right.$ a). Let $g(y):=\gamma_{x_{0}, y-y_{0}}(1)$ for $y \in f(A)$. Then $g(f(x)) \equiv x$ for all $x \in A$, showing that $\left.f\right|_{A}$ is invertible with $g$ as its inverse. To see that $f(A)$ is star-shaped with respect to $y_{0}$, let $x \in A, t \in[0,1]$. Then $y_{0}+$ $t\left(f(x)-y_{0}\right)=f\left(\gamma_{x_{0}, f(x)-y_{0}}(t)\right) \in f(A)$.

Let $\mathcal{A}$ be the subset of $\Omega$ spanned by the maximal liftings, starting from $x_{0}$, of the radii departing from $y_{0}$ in all directions. Then $\mathcal{A}$ is the largest set satisfying the conditions of the previous lemma.

Proposition 2.4. The set $\mathcal{A}:=\left\{\gamma_{x_{0}, v}(t) \mid v \in \mathbb{R}^{n}, t \in I_{x_{0}, v}\right\}$ is the largest of all connected subsets $A$ of $\Omega$, containing $x_{0}$, for which the restriction of $f$ to $A$ is one-to-one and $f(A)$ is star-shaped with respect to $y_{0}$. Moreover, $\mathcal{A}$ is open in $\Omega, 1 \in I_{x_{0}, f(x)-y_{0}}$ for all $x \in \mathcal{A}$ and the inverse of $\left.f\right|_{\mathcal{A}}$ is $y \mapsto \gamma_{x_{0}, y-y_{0}}(1)$.

Proof. To start with, note that we can restrict the times $t$ in the definition of $\mathcal{A}$ to be $>0$, because time 0 corresponds to $x_{0}$, which can be recovered as $x_{0}=\gamma_{x_{0}, 0}(1)$, while reversing the sign of $v$ takes care of the negative times, since $\gamma_{x_{0}, v}(-t)=\gamma_{x_{0},-v}(t)$. The set $\mathcal{A}$ is open because it can be written as the union of a family of sets,

$$
\mathcal{A}=\bigcup_{t>0}\left\{\gamma_{x, v}(t) \mid v \in \mathbb{R}^{n} \text { is such that } t \in I_{x, v}\right\},
$$

all of which are open because they are the images of the open sets $\{v \in$ $\left.\mathbb{R}^{n} \mid t \in I_{x, v}\right\}$ under the open mappings $v \mapsto \gamma_{x, v}(t)$ (Proposition 2.1). $\mathcal{A}$ is also connected because any of its points $\gamma_{x, v}(t)$ is joined to $x_{0}$ by the continuous path $\tau \mapsto \gamma_{x, v}(\tau), \tau \in[0, t]$, that lies entirely in $\mathcal{A}$. It is obvious that $f(\mathcal{A})$ is star-shaped with respect to $y_{0}$. To see that $f$ is one-to-one on $\mathcal{A}$ just note that if $f\left(\gamma_{x_{0}, v}(t)\right)=f\left(\gamma_{x_{0}, v^{\prime}}\left(t^{\prime}\right)\right)$ then $y_{0}+t v=y_{0}+t^{\prime} v^{\prime}$ and, by Proposition 2.1, $\gamma_{x_{0}, v}(t)=\gamma_{x_{0}, v^{\prime}}\left(t^{\prime}\right)$. The fact that $1 \in I_{x_{0}, f(x)-y_{0}}$ for all $x \in \mathcal{A}$ and the formula for the inverse of $\left.f\right|_{\mathcal{A}}$ is a consequence of Lemma 2.3.

Let $A$ be any other subset of $\Omega$ to which the conditions of Lemma 2.3 apply and let $x \in A$. Then $1 \in I_{x_{0}, f(x)-y_{0}}$ and $x=\gamma_{x_{0}, f(x)-y_{0}}(1)$, so that $x \in \mathcal{A}$ as well.

The set $\mathcal{A}$ has a neat interpretation in terms of dynamical systems theory. To see this, let us start from the following (global) dynamical system in $\mathbb{R}^{n}$ :

$$
\Psi: \mathbb{R}^{n} \times \mathbb{R} \rightarrow \mathbb{R}^{n}, \quad \Psi(y, t):=y_{0}+e^{-t}\left(y-y_{0}\right),
$$

whose trajectories are the half-lines hinged at $y_{0}$, but this time they start at $y$ $(\Psi(y, 0)=y)$ and they shoot towards $y_{0}$ with a decreasing exponential parameter instead of a linear one, so that in particular $\Psi(y, t) \rightarrow y_{0}$ as $t \rightarrow \infty$. It is indeed a dynamical system, because $\Psi\left(\Psi\left(y, t_{1}\right), t_{2}\right)=\Psi\left(y, t_{1}+t_{2}\right)$. 
Through the local homeomorphism $f$, this $\Psi$ can be "lifted" to a local dynamical system on $\Omega$, that is to say, there exists an open subset $D_{\Phi}$ of $\Omega \times \mathbb{R}$ and a continuous function $\Phi: D_{\Phi} \rightarrow \Omega$ satisfying

- for all $x \in \Omega$, the set $\left\{t \in \mathbb{R} \mid(x, t) \in D_{\Phi}\right\}$ is an interval containing 0 ,

- $\Phi(x, 0)=x$ for all $x \in \Omega$,

- $\left(x, t_{1}\right),\left(x, t_{1}+t_{2}\right) \in D_{\Phi} \Rightarrow\left(\Phi\left(x, t_{1}\right), t_{2}\right) \in D_{\Phi}$ and $\Phi\left(\Phi\left(x, t_{1}\right), t_{2}\right)=\Phi\left(x, t_{1}+t_{2}\right)$ for all $x \in \Omega, t_{1}, t_{2} \in \mathbb{R}$,

- $f(\Phi(x, t))=\Psi(f(x), t)=y_{0}+e^{-t}\left(f(x)-y_{0}\right)$ for all $(x, t) \in D_{\Phi}$.

In terms of the path-lifting notation that we have already introduced, the maximal dynamical system with such properties is given by

$$
\begin{gathered}
D_{\Phi}:=\left\{(x, t) \in \Omega \times \mathbb{R} \mid\left(x, y_{0}-f(x), 1-e^{-t}\right) \in D\right\}, \\
\Phi(x, t):=\gamma_{x, y_{0}-f(x)}\left(1-e^{-t}\right) .
\end{gathered}
$$

In the particular case when $f$ is a local $C^{1}$ diffeomorphism, $\Phi$ is the flow of the autonomous ordinary differential system

$$
\dot{x}(t)=-f^{\prime}(x(t))^{-1}\left(f(x(t))-f\left(x_{0}\right)\right),
$$

which was first considered by one of the authors in [26].

The usual nomenclature of dynamical systems theory can now be applied to translate topological properties of $f$ into dynamical properties of $\Phi$. The fact that $f$ is a local homeomorphism makes it possible to define $\Phi$, in the first place, but it implies more.

Proposition 2.5. The set $\left\{x_{0}\right\}$ is asymptotically stable for $\Phi$ and $\mathcal{A}$ is its basin of attraction.

Pro of. Remind that a set is asymptotically stable for a (local) dynamical system if it is an attractor and it is stable. Let $U$ be an open neighbourhood of $x_{0}$ where $f$ is one-to-one and let $g:=\left(\left.f\right|_{U}\right)^{-1}$. For any small $r>0$, the ball $B_{r}\left(y_{0}\right)$ is contained in $f(U)$, and for such $r$ let $U_{r}:=g^{-1}\left(B_{r}\left(y_{0}\right)\right)$. Then $U_{r}$ is a neighbourhood of $x_{0}$, and for all $x \in U_{r}$ the trajectories $t \mapsto \Phi(x, t)$ of $\Phi$ are defined globally in the future, belong to $U_{r}$ for all $t \geq 0$ and converge to $x_{0}$ as $t \rightarrow \infty$. Then $\left\{x_{0}\right\}$ is an attractor because it attracts a whole neighbourhood (any $U_{r}$ will do), and it is stable because any of its neighbourhoods contains a positively invariant neighbourhood (again $U_{r}$, with small enough $r$ ) with global existence in the future. Finally, $\mathcal{A}$ is the basin of attraction of $\left\{x_{0}\right\}$ because it is by definition the set of points of $\Omega$ that are attracted to $x_{0}$ by $\Phi$.

Here is now the dynamical systems version of Theorem 1.1.

Proposition 2.6. The following conditions are equivalent: 
$(\alpha) f$ is one-to-one and $f(\Omega)$ is star-shaped with respect to $y_{0}$;

$(\beta)$ the set $\left\{x_{0}\right\}$ is globally asymptotically stable for the local dynamical system $\Phi$, i.e., $\mathcal{A}=\Omega$;

$(\gamma)$ there exists a global Lyapunov function for $\Phi$, in the following sense: a continuous function $k: \Omega \rightarrow \mathbb{R}$ which is proper and bounded from below and such that the composition $t \mapsto k(\Phi(x, t))$ is weakly decreasing for all $x \in \Omega$.

A Lyapunov function $k$ in the sense of $(\gamma)$ has in general only a weak minimum at $x_{0}$ and the monotonicity along the flow is not necessarily strict; if strict minimum and monotonicity were somehow required, it would be enough to replace $k(x)$ with $k(x)+\left|f(x)-y_{0}\right|^{2}$.

We will not insist any further on the dynamical systems interpretation and in particular we will not try to prove Theorem 1.1 as Proposition 2.6. One reason is that we are not aware of a published theorem that would provide the equivalence $(\beta) \Leftrightarrow(\gamma)$ in our precise setting without any hassle. Theorem 2.9 of [4] shows that global asymptotic stability is equivalent to existence of a global Lyapunov function, but it is stated and proved for systems which are globally defined in time. We may think of bridging the gap with a globalizing reparameterization, but that would be a technicality extraneous to the spirit of the present work, and anyway it does not seem to be readily available in the literature except for systems that are associated with autonomous ordinary differential equations (equations (2.8) in our case). The other reason is that $(\mathrm{b}) \Rightarrow(\mathrm{a})$ of Theorem 1.1 is already simple enough to prove directly, while the converse implication gains in geometrical insight and regularity of $k$ if the proof exploits the star-shaped structure of the problem at hand.

The next three propositions are of little intrinsic interest and they will only be used in some proofs in Section 4.

Proposition 2.7. Let $C$ be a compact, connected subset of $\Omega$ containing $x_{0}$. Then the following two conditions are equivalent:

(a) $C \subset \mathcal{A}$ and $f(C)$ is star-shaped with respect to $y_{0}$;

(b) for all $v \in \mathbb{R}^{n}$ the set $\gamma_{x_{0}, v}^{-1}(C)$ is an interval of $\mathbb{R}$.

Pr o of. (a) $\Rightarrow(\mathrm{b})$. Since $C \subset \mathcal{A}, f$ is one-to-one on $C$. Let $v \in \mathbb{R}^{n}$. Since $f(C)$ is star-shaped with respect to $y_{0}$, the set $\gamma_{x_{0}, v}^{-1}=\left\{t \in \mathbb{R} \mid y_{0}+t v \in\right.$ $f(C)\}$ is an interval.

(b) $\Rightarrow($ a). The set $C \cap \mathcal{A}$ is open in $C$. To prove that it is also closed, let $x_{n}=\gamma_{x_{0}, f\left(x_{n}\right)-y_{0}}(1)$ be a sequence of points in $C \cap \mathcal{A}$ converging to $x \in C$. Consider the path $\gamma_{x_{0}, f(x)-y_{0}}$. Applying Proposition 2.2 to the compact set $C$, we find a time $\bar{t}>0$ such that $\gamma_{x_{0}, f(x)-y_{0}}(\bar{t}) \in \Omega \backslash C$. For all $n$ large enough, we will have $\bar{t} \in I_{x_{0}, f\left(x_{n}\right)-y_{0}}$ and also $\gamma_{x_{0}, f\left(x_{n}\right)-y_{0}}(\bar{t}) \in \Omega \backslash C$. But the set of times when $\gamma_{x_{0}, f\left(x_{n}\right)-y_{0}}$ lies in $C$ is an interval by assumption (b). 
Hence $\gamma_{x_{0}, f\left(x_{n}\right)-y_{0}}(t) \in \Omega \backslash C$ for all $t \geq \bar{t}$ and all large $n$, which implies that $\bar{t}>1$, because $\gamma_{x_{0}, f\left(x_{n}\right)-y_{0}}(1) \in C$. We can now write

$$
C \ni x=\lim _{n \rightarrow \infty} x_{n}=\lim _{n \rightarrow \infty} \gamma_{x_{0}, f\left(x_{n}\right)-y_{0}}(1)=\gamma_{x_{0}, f(x)-y_{0}}(1) \in \mathcal{A},
$$

which concludes the proof that $C \subset \mathcal{A}$. The fact that $f(C)$ is star-shaped with respect to $y_{0}$ comes now from Lemma 2.3.

Proposition 2.8. We have $f(\mathcal{A})=f(\Omega)$ if and only if $\mathcal{A}=\Omega$.

Proof. The "if" part is trivial. Also the converse needs very little of the structure of the problem. Suppose that $f(\mathcal{A})=f(\Omega)$. Since $\mathcal{A}$ is open, we will conclude that $\mathcal{A}=\Omega$ if we prove that $\mathcal{A}$ is also closed in $\Omega$. Let then $x_{n} \in \mathcal{A}$ be a sequence converging to $x \in \Omega$. Since $f(\mathcal{A})=f(\Omega)$, there exists $\bar{x} \in \mathcal{A}$ such that $f(\bar{x})=f(x)$. Recalling that $\left.f\right|_{\mathcal{A}}: \mathcal{A} \rightarrow f(\mathcal{A})$ is a homeomorphism, from $f\left(x_{n}\right) \rightarrow f(\bar{x})$ we deduce $x_{n} \rightarrow \bar{x}$, whence $\bar{x}=$ $x \in \mathcal{A}$.

Proposition 2.9. Let $\Omega$ be a nonempty, open and connected subset of $\mathbb{R}^{n}$, and $f: \Omega \rightarrow \mathbb{R}^{n}$ be a local homeomorphism. The following two conditions are equivalent:

(a) $f$ is one-to-one and $f(\Omega)=\mathbb{R}^{n}$;

(b) there exist a continuous, proper function $k: \Omega \rightarrow \mathbb{R}$ bounded from below, a locally bounded function $\varphi:\left[0, \infty\left[\rightarrow \mathbb{R}\right.\right.$ and a point $x_{0} \in \Omega$ such that

$$
k(x) \leq \varphi\left(\left|f(x)-f\left(x_{0}\right)\right|\right) \quad \text { for all } x \in \mathcal{A},
$$

where $\mathcal{A}=\mathcal{A}_{x_{0}}$ is the set defined in Proposition 2.4.

Proof. If (a) holds, then for any $x_{0} \in \Omega$ we can define $k(x):=\mid f(x)-$ $f\left(x_{0}\right) \mid$ and $\varphi(r):=r$.

Conversely, let $y \in \mathbb{R}^{n}$ be arbitrary and $y_{0}:=f\left(x_{0}\right)$. Consider the path $\gamma:=\gamma_{x_{0}, y-y_{0}}$, that starts from $x_{0}$ and whose image through $f$ is a segment of the straight line originating at $y_{0}$ and pointing toward $y$. All of $\gamma$ is contained in $\mathcal{A}$, of course. For times $t \in I_{x_{0}, y-y_{0}} \cap[0,1]$ we have

$$
\begin{aligned}
k(\gamma(t)) & \leq \varphi\left(\left|f(\gamma(t))-y_{0}\right|\right)=\varphi\left(t\left|y-y_{0}\right|\right) \\
& \leq \sup \left\{\varphi(r)|0 \leq r \leq| y-y_{0} \mid\right\},
\end{aligned}
$$

so that for all those times the path lies in the compact set $C:=$ $k^{-1}\left(\left[\inf k, \sup _{\left[0,\left|y-y_{0}\right|\right]} \varphi\right]\right)$. Because of Proposition 2.2, there exists a time $t_{0}>0$ for which $\gamma\left(t_{0}\right) \in \Omega \backslash C$. Necessarily then $t_{0}>1$, and

$$
y=f(\gamma(1)) \in f(\mathcal{A}) .
$$

This shows that $f(\mathcal{A})=\mathbb{R}^{n}$ and the conclusion follows from Proposition 2.8.

The following simple consequence when $\Omega=\mathbb{R}^{n}$ is well-known: 
Corollary 2.10. A local homeomorphism $f: \mathbb{R}^{n} \rightarrow \mathbb{R}^{n}$ is one-to-one and onto $\mathbb{R}^{n}$ if and only if $\lim _{|x| \rightarrow \infty}|f(x)|=\infty$, that is to say, if and only if $f$ is a proper map.

Proof. For the "if" part, apply Proposition 2.9 above with $x_{0}:=0$, $k(x):=|f(x)-f(0)|$ and $\varphi(r):=r$.

One more easy lemma before wrapping up the first half of the proof of Theorem 1.1.

Lemma 2.11. Let $k: \Omega \rightarrow \mathbb{R}$ be a function such that, for any local inverse $g$ of $f$ and any $v \in \mathbb{R}^{n}$ the real function $t \mapsto k\left(g\left(y_{0}+t v\right)\right)$ is weakly increasing in all the $t \geq 0$ where it is defined. Then the function $t \mapsto k\left(\gamma_{x_{0}, v}(t)\right)$ is weakly increasing on $I_{x_{0}, v} \cap\{t \mid t \geq 0\}$.

Proof of Theorem 1.1, (b) $\Rightarrow(a)$. Assume condition (b) of Theorem 1.1. Verifying condition (a) amounts to proving that $\mathcal{A}=\Omega$, where $\mathcal{A}$ is the set defined in Proposition 2.4. Since $\mathcal{A}$ is nonempty and open, all we are left to do is to verify that it is also closed in $\Omega$. Let $z_{n}=\gamma_{x_{0}, f\left(z_{n}\right)-y_{0}}(1)$ be a sequence of points of $\mathcal{A}$ converging to $z \in \Omega$. The task is to show that $z \in \mathcal{A}$. Let $M$ be the (finite) supremum of the continuous function $k$ on the compact set $\{z\} \cup\left\{z_{n} \mid n \in \mathbb{N}\right\}$. Since $k$ is also a proper map, the set

$$
C:=k^{-1}([\inf k, M])
$$

is compact. By Proposition 2.2 the path $\gamma_{x_{0}, f(z)-y_{0}}$ will be outside $C$ starting from a certain time $\bar{t} \geq 0$, that is,

$$
k\left(\gamma_{x_{0}, f(z)-y_{0}}(t)\right)>M \quad \text { for all } t \geq \bar{t} .
$$

For all indices $n$ large enough, the paths $\gamma_{x_{0}, f\left(z_{n}\right)-y_{0}}$ will also be defined up to time $\bar{t}$, and, using again the continuity of $k$,

$$
k\left(\gamma_{x_{0}, f\left(z_{n}\right)-y_{0}}(\bar{t})\right)>M \quad \text { for all large } n .
$$

But those paths are also defined up to $t=1$, and along them the function $k$ is weakly increasing (Lemma 2.11) for nonnegative times. Hence

$$
\begin{array}{r}
k\left(\gamma_{x_{0}, f\left(z_{n}\right)-y_{0}}(t)\right) \leq k\left(\gamma_{x_{0}, f\left(z_{n}\right)-y_{0}}(1)\right)=k\left(z_{n}\right) \leq M \\
\text { for all } t \in[0,1] \text { and all large } n .
\end{array}
$$

By comparing inequalities (2.15) and (2.16) we see that $\bar{t}$ is necessarily larger than 1. In particular, the path $\gamma_{x_{0}, f(z)-y_{0}}$ is defined at $t=1$ and

$$
z=\lim _{n \rightarrow \infty} z_{n}=\lim _{n \rightarrow \infty} \gamma_{x_{0}, f\left(z_{n}\right)-y_{0}}(1)=\gamma_{x_{0}, f(z)-y_{0}}(1) \in \mathcal{A} .
$$

R e mark 2.12. Condition (b) of Theorem 1.1 can be relaxed as follows:

(b)' there exists a continuous function $\widetilde{k}:\left\{x \in \Omega \mid f(x) \neq y_{0}\right\} \rightarrow \mathbb{R}$ for which the set

$$
\left\{x \in \Omega|\widetilde{k}(x) \leq a,| f(x)-y_{0} \mid \geq \varepsilon\right\}
$$


is compact for any $a \in \mathbb{R}, \varepsilon>0$, and such that, for every local inverse $g$ of $f$ and any vector $v \in \mathbb{R}^{n} \backslash\{0\}$, the real function

$$
t \mapsto \widetilde{k}\left(g\left(y_{0}+t v\right)\right)
$$

is locally weakly increasing at all the $t>0$ where it is defined.

Proof. As in the proof of Theorem 1.1 above, let $z_{n}=\gamma_{x_{0}, f\left(z_{n}\right)-y_{0}}(1)$ be a sequence of points in $\mathcal{A}$ converging to $z \in \Omega$. We distinguish two cases according to whether $f(z)=y_{0}$ or $f(z) \neq y_{0}$.

First case: $f(z)=y_{0}$. Since $\left.f\right|_{\mathcal{A}}: \mathcal{A} \rightarrow f(\mathcal{A})$ is a homeomorphism, the relation $f\left(z_{n}\right) \rightarrow f\left(x_{0}\right)=y_{0}=f(z)$ implies that $z_{n} \rightarrow x_{0}$, so that $z=x_{0} \in \mathcal{A}$.

Second case: $f(z) \neq y_{0}$. Then we can assume that $\left|f\left(z_{n}\right)-y_{0}\right| \geq \varepsilon$ for some $\varepsilon>0$ and all $n$. From this point on we can proceed exactly as in the proof of Theorem 1.1, with the only provision of setting

$$
C:=\left\{x \in \Omega|\widetilde{k}(x) \leq M,| f(x)-y_{0} \mid \geq \varepsilon\right\}
$$

instead of (2.13).

3. Proof of Theorem 1.1, (a) $\Rightarrow(\mathrm{b})$. Suppose that a homeomorphism $f: \Omega \rightarrow \Delta$ is given between two nonempty, open subsets of $\mathbb{R}^{n}$, of which $\Delta$ is star-shaped with respect to one of its points $y_{0}$. The problem is to prove the existence of a continuous, proper function $k: \Omega \rightarrow \mathbb{R}$ bounded from below such that the composite function $k \circ f^{-1}: \Delta \rightarrow \mathbb{R}$ is weakly increasing along the radii departing from $y_{0}$.

An easy special case, to start with: the function

$$
k: x \mapsto\left|f(x)-y_{0}\right|
$$

is trivially continuous, bounded from below and its composition with $f^{-1}$ is weakly increasing along the radii departing from $y_{0}$. Unfortunately, it solves the problem (i.e., it is proper) if and only if $\Delta=\mathbb{R}^{n}$ : in fact, for any $r \geq 0$, the closed ball $\bar{B}_{r}\left(y_{0}\right):=\left\{y \in \mathbb{R}^{n}|| y-y_{0} \mid \leq r\right\}$ is compact and connected, so that its open subset $f(\{x \in \Omega \mid 0 \leq k(x) \leq r\})=\Delta \cap \bar{B}_{r}\left(y_{0}\right)$ is compact if and only if it is closed, that is to say, if and only if it coincides with the whole of $\bar{B}_{r}\left(y_{0}\right)$. Other particular shapes of $\Delta$ (open balls, for example) can be tackled with ad hoc auxiliary functions $k$. In the rest of this section we will treat the most general case, except for the somewhat simplifying assumption that $\Delta \neq \mathbb{R}^{n}$.

A few more simplifications of the problem come cheaply. The first one is to suppose $y_{0}$ to be the origin of $\mathbb{R}^{n}$. In the rest of this section all sets that will be described as star-shaped will be meant to be so with respect to the origin. Next, we see that it suffices to assign a continuous, proper, bounded below and radially weakly increasing function $K_{\Delta}: \Delta \rightarrow \mathbb{R}$ to 
any open, star-shaped subset $\Delta$ of $\mathbb{R}^{n}$, i.e., a solution to the special case when $f$ is the identity mapping on $\Omega \equiv \Delta$ : in fact, the solution of the general case can be recovered as $k:=K_{\Delta} \circ f$. Moreover, we can concentrate the search on the functions $K_{\Delta}$ whose radial monotonicity is strict, because from any solution $K_{\Delta}$ we can define the radially strictly increasing $\widetilde{K}_{\Delta}(y):=$ $K_{\Delta}(y)+|y|$, which is easily seen to be also continuous, proper and bounded from below. Finally, it does no harm to require that $K_{\Delta}$ vanish in the origin (whence $K_{\Delta}(y)>0$ for $y \in \Delta \backslash\{0\}$ ).

A function $K_{\Delta}$ that happened to comply with all the above rules will also provide a solution to the well-known (see [24], Vol. 1, p. 322) problem of proving that an open, star-shaped subset $\Delta$ of $\mathbb{R}^{n}$ is homeomorphic to the whole of $\mathbb{R}^{n}$, since the mapping

$$
y \mapsto \begin{cases}0 & \text { if } y=0, \\ \frac{K_{\Delta}(y)}{|y|} y & \text { if } y \in \Delta \backslash\{0\}\end{cases}
$$

will do the job. An arbitrary homeomorphism $\varphi: \Delta \rightarrow \mathbb{R}^{n}$ will not return us a suitable function $K_{\Delta}$, though, unless $\varphi$ leaves the half-lines radiating from the origin invariant. Since the reference [24] gives no existence proofs of homeomorphisms, much the less of ones with the additional properties that we need here, we feel we can not exempt ourselves from providing the reader with a very detailed construction of the function $K_{\Delta}$.

The level sets $\left\{y \in \Delta \mid K_{\Delta}(y)=c\right\}, c>0$, of our function $K_{\Delta}$ are far from being arbitrary subsets of $\Delta$ : they are compact and they are the boundaries of open, star-shaped sets, namely, of $\left\{y \in \Delta \mid K_{\Delta}(y)<c\right\}$. This geometric property of the level sets of $K_{\Delta}$ is what underlies our construction: we will define a nested, increasing family $\Delta_{c}, c>0$, of bounded subsets of $\Delta$, in such a way that their boundaries $\partial \Delta_{c}$ will be a partition of $\Delta \backslash\{0\}$ and that the function defined as

$$
K_{\Delta}(y):= \begin{cases}0 & \text { if } y=0, \\ c & \text { if } c>0 \text { and } y \in \partial \Delta_{c}\end{cases}
$$

will be what we need.

Let $S:=\left\{u \in \mathbb{R}^{n}|| u \mid=1\right\}$ be the unit sphere of $\mathbb{R}^{n}$, viewed, case by case, either as the set of directions in $\mathbb{R}^{n}$, or as a topological or metric subspace of $\mathbb{R}^{n}$. With an arbitrary subset $\Sigma$ of $\mathbb{R}^{n}$ we can associate the following extended-real-valued function on $S$ :

$$
\varrho_{\Sigma}: S \rightarrow[0, \infty], \quad \varrho_{\Sigma}(u):=\sup \{\lambda \geq 0 \mid \lambda u \in \Sigma\},
$$

that assigns to any $u \in S$ the minimum distance beyond which no more points of $\Sigma$ can be found in the direction $u$. The set $\left\{\varrho_{\Sigma}(u) u \mid u \in S, \varrho_{\Sigma}(u)<\right.$ $\infty\}$ is always contained in the boundary of $\Sigma$. Of course, the function $\varrho_{\Sigma}$ is specially well-suited to handle star-shaped sets, in which case it deserves 
the name of radial function. $\Sigma$ is star-shaped if and only if $\Sigma \supset\{\lambda u \mid u \in S$, $\left.0 \leq \lambda<\varrho_{\Sigma}(u)\right\}$. Some relationships between topological properties of $\Sigma$ and $\varrho_{\Sigma}$ are stated in the proposition below. We denote by int $A$ the interior of a subset $A$ of $\mathbb{R}^{n}$ and talk of continuity or semicontinuity of $\varrho_{\Sigma}$ in the extended-real-valued function sense.

Proposition 3.1. Let $\Sigma$ be a star-shaped subset of $\mathbb{R}^{n}$ and let $\bar{u} \in S$. Then

1. $\left\{\lambda \bar{u} \mid 0<\lambda<\varrho_{\Sigma}(\bar{u})\right\} \subset$ int $\Sigma$ if and only if $\varrho_{\Sigma}$ is lower semicontinuous at $\bar{u}$;

2. $\left\{\lambda \bar{u} \mid \varrho_{\Sigma}(\bar{u})<\lambda<\infty\right\} \subset \operatorname{int}\left(\mathbb{R}^{n} \backslash \Sigma\right)$ if and only if $\varrho_{\Sigma}$ is upper semicontinuous at $\bar{u}$;

3. $\left\{\lambda \bar{u} \mid 0<\lambda=\varrho_{\Sigma}(\bar{u})<\infty\right\}=\partial \Sigma \cap\{\lambda \bar{u} \mid \lambda>0\}$ if and only if $\varrho_{\Sigma}$ is continuous at $\bar{u}$.

Proof. Suppose $\left\{\lambda \bar{u} \mid 0<\lambda<\varrho_{\Sigma}(\bar{u})\right\} \subset$ int $\Sigma$. If $\varrho_{\Sigma}(\bar{u})=0$ it is obvious that $\varrho_{\Sigma}$ is lower semicontinuous at $\bar{u}$, because $\varrho_{\Sigma} \geq 0$. If $\varrho_{\Sigma}(\bar{u})>0$ then let $0<M<\varrho_{\Sigma}(\bar{u}) \leq \infty$. Since $M \bar{u} \in \Sigma$, there exists $\varepsilon>0$ such that

$$
\forall y \in \mathbb{R}^{n} \quad|y-M \bar{u}|<\varepsilon \Rightarrow y \in \Sigma .
$$

With the change of variable $M x=y$ we can write

$$
\forall x \in \mathbb{R}^{n} \quad|x-\bar{u}|<\varepsilon / M \Rightarrow M x \in \Sigma,
$$

whence, restricting to $x=u \in S$ and recalling the definition of the radial function,

$$
\forall u \in S \quad|u-\bar{u}|<\varepsilon / M \Rightarrow \varrho_{\Sigma}(u) \geq M,
$$

which shows that $\varrho_{\Sigma}$ is lower semicontinuous at $\bar{u}$.

Conversely, suppose that $\varrho_{\Sigma}$ is lower semicontinuous at $\bar{u}$. If $\varrho_{\Sigma}(\bar{u})=0$ then the inclusion $\left\{\lambda \bar{u} \mid 0<\lambda<\varrho_{\Sigma}(\bar{u})\right\}=\emptyset \subset$ int $\Sigma$ is obvious. If $\varrho_{\Sigma}(\bar{u})>$ 0 then the function

$$
x \mapsto \varrho_{\Sigma}(x /|x|)-|x|, \quad \mathbb{R}^{n} \backslash\{0\} \rightarrow \mathbb{R} \cup\{\infty\},
$$

is lower semicontinuous and takes the strictly positive value $\varrho_{\Sigma}(\bar{u})-\lambda$ at any point of the form $\lambda \bar{u}$ with $0<\lambda<\varrho_{\Sigma}(\bar{u})$. Hence it is positive in a whole neighbourhood of each of these points. But, for $x \neq 0, \varrho_{\Sigma}(x /|x|)-|x|>0$ implies $x \in \Sigma$ because $\Sigma$ is star-shaped. Hence the inclusion $\{\lambda \bar{u} \mid 0<\lambda<$ $\left.\varrho_{\Sigma}(\bar{u})\right\} \subset$ int $\Sigma$ holds as desired.

Part 2 is analogous and part 3 is simply the conjunction of parts 1 and 2 .

Corollary 3.2. For a star-shaped subset $\Sigma$ of $\mathbb{R}^{n}$, the following two conditions are equivalent:

I. $\Sigma$ is open; 
II. $\varrho_{\Sigma}$ is lower semicontinuous on $S$, inf $\varrho_{\Sigma}>0$ and $\Sigma=\{\lambda u \mid u \in$ $\left.S, \lambda \in \mathbb{R}, 0 \leq \lambda<\varrho_{\Sigma}(u)\right\}$.

COROLlary 3.3. For a star-shaped subset $\Sigma$ of $\mathbb{R}^{n}$, the following two conditions are equivalent:

III. $\partial \Sigma=\left\{\varrho_{\Sigma}(u) u \mid u \in S, \varrho_{\Sigma}(u)<\infty\right\}$;

IV. $\varrho_{\Sigma}$ is continuous (as an extended-real-valued function on $S$ ).

The radial function of an open, star-shaped set need not be continuous, as can be seen from the example $\mathbb{R}^{2} \backslash\{(\lambda, 0) \mid \lambda \geq 1\}$.

Let us turn back to the open, star-shaped set $\Delta$ where we started from. This is completely determined by its radial function $\varrho_{\Delta}$. It is just natural to expect that any other function defined on $\Delta$ that only depends on the geometry of $\Delta$ in its quality of star-shaped set will be best obtained by manipulating $\varrho_{\Delta}$.

A family $\Delta_{c}, c>0$, of star-shaped sets defines the function

$$
\varrho:] 0, \infty\left[\times S \rightarrow[0, \infty], \quad \varrho(c, u):=\varrho_{\Delta_{c}}(u) .\right.
$$

If the sets $\Delta_{c}$ are open, then they are contained in $\Delta$ if and only if $\varrho(c, u) \leq$ $\varrho_{\Delta}(u)$ for all $c, u$. The nesting $\Delta_{c_{1}} \subset \Delta_{c_{2}}$ translates into the inequality $\varrho\left(c_{1}, \cdot\right) \leq \varrho\left(c_{2}, \cdot\right)$ and a necessary condition for $\partial \Delta_{c_{1}}$ to be disjoint from $\partial \Delta_{c_{2}}$ is for the inequality to be strict, a condition that becomes sufficient if $\varrho(c, u)$ is continuous in $u$ for every fixed $c$, because of Proposition 3.1. If we assume $\varrho(c, u)$ to be strictly increasing in $c$ and continuous in $u$, then the boundaries $\partial \Delta_{c}$ will cover $\Delta \backslash\{0\}$ if and only if $\varrho(\cdot, u)$ spans the whole interval $] 0, \varrho_{\Delta}(u)[$, i.e., if it is continuous, $\inf \varrho(\cdot, u)=0$ and $\sup \varrho(\cdot, u)=\varrho \Delta(u)$ for all fixed $u \in S$.

A function $\varrho(c, u)$ that fulfills all these wishes is easy to define under the additional hypothesis that $\varrho_{\Delta}$ be continuous and bounded. In fact, we may simply set

$$
\varrho(c, u):=\frac{c}{c+1} \varrho_{\Delta}(u) \quad \text { for } c>0, u \in S .
$$

The function $K_{\Delta}$ corresponding to this choice of $\varrho$, according to our program, is, outside the origin:

$$
\begin{aligned}
K_{\Delta}(y)=c & \Leftrightarrow y \in \partial \Delta_{c} \Leftrightarrow(y=\varrho(c, u) u \text { with } u \in S) \\
& \Leftrightarrow|y|=\varrho(c, y /|y|)=\frac{c}{c+1} \varrho \Delta(y /|y|) \\
& \Leftrightarrow K_{\Delta}(y)=\frac{|y|}{\varrho_{\Delta}(y /|y|)-|y|} .
\end{aligned}
$$

It is easy to verify that this $K_{\Delta}$ is indeed continuous, proper, nonnegative, vanishing at the origin and radially strictly increasing. When $\Delta$ is convex, then this $K_{\Delta}$ is also a convex function. 
The construction that we are going to provide for the general case when $\varrho_{\Delta}$ is not assumed to be continuous and bounded is more elaborate but will have the side advantage of showing that more regularity can be asked of $K_{\Delta}$ than simple continuity.

The function $\widetilde{\varrho}$ defined as

$$
\widetilde{\varrho}:] 0, \infty\left[\times S \rightarrow\left[0, \infty\left[, \quad \widetilde{\varrho}(c, u):=\inf _{v \in S}\left(\varrho_{\Delta}(v)+c|u-v|\right),\right.\right.\right.
$$

is nonnegative and finite because $\varrho_{\Delta}$ is nonnegative and not identically $\infty$ (this is the single spot where we use the assumption that $\Delta \neq \mathbb{R}^{n}$ ). The following inequalities are immediate, taking into account that $|u-v| \leq 2$ for $u, v \in S$ :

$$
\begin{gathered}
0<\inf \varrho_{\Delta} \leq \widetilde{\varrho}(c, u) \leq \inf \varrho_{\Delta}+2 c<\infty, \\
\widetilde{\varrho}(c, u) \leq \varrho_{\Delta}(u) \quad \text { for all } c>0, u \in S .
\end{gathered}
$$

The function $\widetilde{\varrho}$ can be described as the pointwise infimum of the family of functions $(c, u) \mapsto \varrho_{\Delta}(v)+c|u-v|$, indexed by those $v \in S$ for which $\varrho_{\Delta}(v)<\infty$. Each function in the family is Lipschitz continuous with respect to $c$ with constant 2, and it is also weakly increasing in $c$, for every fixed $u \in$ $S$; with respect to $u$ it is Lipschitz continuous with constant $c$ (here $S$ is seen as a metric subspace of $\mathbb{R}^{n}$ ). These are the kind of properties that transfer without trouble to the pointwise infimum, if only it is finite, as is the case right here:

$$
\begin{aligned}
& \left|\widetilde{\varrho}\left(c_{1}, u_{1}\right)-\widetilde{\varrho}\left(c_{2}, u_{2}\right)\right| \leq 2\left|c_{1}-c_{2}\right|+\max \left\{c_{1}, c_{2}\right\}\left|u_{1}-u_{2}\right|, \\
& 0<c_{1}<c_{2} \Rightarrow \widetilde{\varrho}\left(c_{1}, u\right) \leq \widetilde{\varrho}\left(c_{2}, u\right) \quad \text { for all } c_{1}, c_{2}>0, u, u_{1}, u_{2} \in S .
\end{aligned}
$$

The function $\widetilde{\varrho}(c, \cdot)$ could be characterized as the largest Lipschitz function on $S$ with constant $c$ which is $\leq \varrho_{\Delta}$.

To prove the next property of $\widetilde{\varrho}$ we will need to exploit the fact that $\Delta$ is open.

Proposition 3.3. $\sup _{c>0} \widetilde{\varrho}(c, u)=\lim _{c \rightarrow \infty} \widetilde{\varrho}(c, u)=\varrho_{\Delta}(u)$ for all $u \in S$.

Proof. Note that there would be no hope to prove the relationship if $\Delta$ were not open, because the pointwise supremum of a family of continuous functions is necessarily lower semicontinuous. Now, given any $\bar{u} \in S$ and $M<\varrho_{\Delta}(\bar{u})$ we can find an $\varepsilon>0$ such that

$$
|v-\bar{u}|<\varepsilon \Rightarrow \varrho_{\Delta}(u) \geq M \quad \text { for any } v \in S .
$$

Then, for all $v \in S$ we can write

$$
\begin{aligned}
& |v-\bar{u}|<\varepsilon \Rightarrow \varrho_{\Delta}(v)+c|v-\bar{u}| \geq M, \\
& |v-\bar{u}| \geq \varepsilon \Rightarrow \varrho_{\Delta}(v)+c|v-\bar{u}| \geq c \varepsilon,
\end{aligned}
$$


so that

$$
\widetilde{\varrho}(c, \bar{u})=\inf _{v \in S}\left(\varrho_{\Delta}(v)+c|v-\bar{u}|\right) \geq M \quad \text { for } c \geq M / \varepsilon,
$$

which concludes the proof.

The function $\widetilde{\varrho}$ is not yet exactly what we wanted, because as a function of $c$ for fixed $u$ it is not strictly increasing (it is constant, for example, at the minimum points of $\varrho_{\Delta}$ ) and its infimum is inf $\varrho_{\Delta}$, which is $>0$ :

$$
\inf \varrho_{\Delta} \leq \inf _{c>0} \widetilde{\varrho}(c, u)=\lim _{c \searrow 0} \widetilde{\varrho}(c, u) \leq \lim _{c \searrow 0}\left(\inf \varrho_{\Delta}+2 c\right)=\inf \varrho_{\Delta} .
$$

These drawbacks can be easily corrected, however, by adding a suitable continuous and strictly increasing function of $c$ that spans $]-\inf \varrho_{\Delta}, 0[$ for $c>0$. Our particular choice is

$$
\varrho:] 0, \infty\left[\rightarrow \mathbb{R}, \quad \varrho(c, u):=\widetilde{\varrho}(c, u)-\frac{\inf \varrho \Delta}{1+c} .\right.
$$

For each $u$ the function $\varrho(\cdot, u)$ now spans $] 0, \varrho_{\Delta}(u)[$ and not only it is strictly increasing but

$$
\begin{aligned}
0<c_{1}<c_{2} \Rightarrow \varrho\left(c_{2}, u\right)-\varrho\left(c_{1}, u\right) & \geq\left(-\frac{1}{1+c_{2}}+\frac{1}{1+c_{1}}\right) \inf \varrho \Delta \\
& \geq \frac{\inf \varrho \Delta}{\left(1+c_{2}\right)^{2}}\left|c_{2}-c_{1}\right| .
\end{aligned}
$$

Since the function $c \mapsto-\left(\inf \varrho_{\Delta}\right) /(1+c)$ is Lipschitz continuous with constant inf $\varrho_{\Delta}$ for $c>0$, the first of the formulas (3.14) becomes

$$
\begin{array}{r}
\left|\varrho\left(c_{1}, u_{1}\right)-\varrho\left(c_{2}, u_{2}\right)\right| \leq(2+\inf \varrho \Delta)\left|c_{1}-c_{2}\right|+\max \left\{c_{1}, c_{2}\right\}\left|u_{1}-u_{2}\right| \\
\text { for all } c_{1}, c_{2}>0, u_{1}, u_{2} \in S .
\end{array}
$$

For any $c>0$ the set $\Delta_{c}:=\{\lambda u \mid u \in S, 0 \leq \lambda<\varrho(c, u)\}$ is open, star-shaped and bounded and the boundaries $\partial \Delta_{c}=\{\varrho(c, u) u \mid u \in S\}$ are a partition of $\Delta \backslash\{0\}$. We can indeed define $K_{\Delta}$ as in our program:

$$
\begin{aligned}
K_{\Delta}(0):=0, & \\
K_{\Delta}(y)=c & \Leftrightarrow y \in \partial \Delta_{c} \Leftrightarrow(y=\varrho(c, u) u \text { with } u \in S) \\
& \Leftrightarrow|y|=\varrho(c, y /|y|) \quad \text { if } y \in \Delta \backslash\{0\} .
\end{aligned}
$$

Since

$$
\varrho(c, u)=\widetilde{\varrho}(c, u)-\frac{\inf \varrho_{\Delta}}{1+c}\left\{\begin{array}{l}
\geq \inf \varrho_{\Delta}-\frac{\inf \varrho_{\Delta}}{1+c}=\frac{c}{1+c} \inf \varrho_{\Delta}, \\
\leq \inf \varrho_{\Delta}+2 c-\frac{\inf \varrho_{\Delta}}{1+c} \leq\left(2+\inf \varrho_{\Delta}\right) c
\end{array}\right.
$$


we have

$$
K_{\Delta}(y) \begin{cases}\leq \frac{|y|}{\inf \varrho_{\Delta}-|y|} & \text { if } y \in \Delta, 0<|y|<\inf \varrho_{\Delta}, \\ \geq \frac{|y|}{2+\inf \varrho_{\Delta}} & \text { if } y \in \Delta,\end{cases}
$$

which shows in particular that $K_{\Delta}$ is continuous at $y=0$. As for the continuity elsewhere, we can write the inequality, for $y, y^{\prime} \in \Delta_{c_{0}} \backslash\{0\}$, $K_{\Delta}(y)=c<c_{0}, K_{\Delta}\left(y^{\prime}\right)=c^{\prime}<c_{0}$,

$$
\begin{aligned}
\left|y-y^{\prime}\right| & \geq|| y|-| y^{\prime}||=\left|\widetilde{\varrho}\left(c, \frac{y}{|y|}\right)-\widetilde{\varrho}\left(c^{\prime}, \frac{y^{\prime}}{\left|y^{\prime}\right|}\right)\right| \\
& \geq\left|\widetilde{\varrho}\left(c, \frac{y}{|y|}\right)-\widetilde{\varrho}\left(c^{\prime}, \frac{y}{|y|}\right)\right|-\left|\widetilde{\varrho}\left(c^{\prime}, \frac{y}{|y|}\right)-\widetilde{\varrho}\left(c^{\prime}, \frac{y^{\prime}}{\left|y^{\prime}\right|}\right)\right| \\
& \geq \frac{\inf \varrho \Delta}{\left(1+c_{0}\right)^{2}}\left|c-c^{\prime}\right|-c^{\prime}\left|\frac{y}{|y|}-\frac{y^{\prime}}{\left|y^{\prime}\right|}\right|
\end{aligned}
$$

whence

$$
\left|K_{\Delta}(y)-K_{\Delta}\left(y^{\prime}\right)\right|=\left|c-c^{\prime}\right| \leq \frac{\left(1+c_{0}\right)^{2}}{\inf \varrho_{\Delta}}\left(\left|y-y^{\prime}\right|+c^{\prime}\left|\frac{y}{|y|}-\frac{y^{\prime}}{\left|y^{\prime}\right|}\right|\right) .
$$

But

$$
\begin{aligned}
\left|\frac{y}{|y|}-\frac{y^{\prime}}{\left|y^{\prime}\right|}\right| & \leq\left|\frac{y}{|y|}-\frac{y}{\left|y^{\prime}\right|}\right|+\left|\frac{y}{\left|y^{\prime}\right|}-\frac{y^{\prime}}{\left|y^{\prime}\right|}\right| \\
& =\frac{|| y^{\prime}|-| y||}{\left|y^{\prime}\right|}+\frac{\left|y-y^{\prime}\right|}{\left|y^{\prime}\right|} \leq \frac{2}{\left|y^{\prime}\right|}\left|y-y^{\prime}\right|,
\end{aligned}
$$

and, from (3.24),

$$
\begin{aligned}
\frac{c^{\prime}}{\left|y^{\prime}\right|} & =\frac{K_{\Delta}\left(y^{\prime}\right)}{\left|y^{\prime}\right|} \\
& \leq \begin{cases}\frac{2 c_{0}}{\inf \varrho_{\Delta}} & \text { if } y^{\prime} \in \Delta_{c_{0}},\left|y^{\prime}\right| \geq \inf \varrho_{\Delta} / 2, \\
\frac{1}{\inf \varrho_{\Delta}-\left|y^{\prime}\right|} \leq \frac{2}{\inf \varrho_{\Delta}} & \text { if } y^{\prime} \in \Delta_{c_{0}}, 0<\left|y_{0}\right|<\inf \varrho_{\Delta} / 2,\end{cases}
\end{aligned}
$$

so that

$$
c^{\prime}\left|\frac{y}{|y|}-\frac{y^{\prime}}{\left|y^{\prime}\right|}\right| \leq \frac{2 c^{\prime}}{\left|y^{\prime}\right|}\left|y-y^{\prime}\right| \leq \frac{4\left(1+c_{0}\right)}{\inf \varrho \Delta}\left|y-y^{\prime}\right|,
$$

and $K_{\Delta}$ is Lipschitz continuous on $\Delta_{c_{0}}$ (the origin is recovered by continuity):

$$
\begin{aligned}
& \left|K_{\Delta}(y)-K_{\Delta}\left(y^{\prime}\right)\right| \leq \frac{4\left(1+c_{0}\right)^{2}\left(4+4 c_{0}+\inf \varrho_{\Delta}\right)}{\left(\inf \varrho_{\Delta}\right)^{2}}\left|y-y^{\prime}\right| \\
& \text { for all } y, y^{\prime} \in \Delta_{c_{0}}
\end{aligned}
$$


Since the sets $\Delta_{c}$ invade $\Delta$, we can say that $K_{\Delta}$ is Lipschitz continuous on compact subsets of $\Delta$; in particular, it is locally Lipschitz continuous.

The last thing to verify is that $K_{\Delta}$ is a proper map: this is easy because the set

$$
\begin{aligned}
K_{\Delta}^{-1}([0, c]) & =\{0\} \cup\{y \in \Delta \backslash\{0\}|| y \mid \leq \varrho(c, y /|y|)\} \\
& =\{\lambda u \mid u \in S, 0 \leq \lambda \leq \varrho(c, u)\}=\Delta_{c} \cup \partial \Delta_{c}
\end{aligned}
$$

is closed and bounded in $\mathbb{R}^{n}$ for any $c>0$.

One may wonder whether $K_{\Delta}$ can be made $C^{\infty}$ differentiable. This is indeed the case: it suffices to discretize the parameter $c$ of the function $\varrho(c, u)$ to integer values $n \in \mathbb{N}$, then to approximate each $\varrho(n, \cdot): S \rightarrow \mathbb{R}$ by a $C^{\infty}$ function by using the Stone-Weierstraß theorem, only taking care of retaining the strict monotonicity and the value of the limit as $c=n \rightarrow \infty$, and finally to restore the real value to $c$ by some kind of $C^{\infty}$ interpolation between the regularized $c$-discretized functions. More pictorially, this amounts to smoothing out the boundaries of the sets $\Delta_{n}$ and then interpolating between each consecutive pair. We will not provide the details of the construction here.

\section{Results for smoother transformations}

Proof of Proposition 1.2. Suppose that equation (1.2) holds. Let $v \in \mathbb{R}^{n}$ and $g$ be a local inverse of $f$ whose domain intersects the half-line $\left\{y_{0}+t v \mid t \geq 0\right\}$. We want to prove that the function $t \mapsto k\left(g\left(y_{0}+t v\right)\right)$ is locally weakly increasing for the $t \geq 0$ where it is defined. The case $v=0$ is trivial and we can suppose $v \neq 0$. Moreover, since $t \mapsto k\left(g\left(y_{0}+t v\right)\right)$ is continuous and defined on an open subset of $\mathbb{R}$, it will be pointwise weakly increasing for $t \geq 0$ if and only if it is for $t>0$. Now, for $t_{0}>0$ we have $g\left(y_{0}+t_{0} v\right) \neq x_{0}$, so that $k$ is differentiable at $x_{1}:=g\left(y_{0}+t_{0} v\right)$ and we can compute

$$
\begin{aligned}
\left.\frac{d}{d t} k\left(g\left(y_{0}+t v\right)\right)\right|_{t=t_{0}} & =k^{\prime}\left(x_{1}\right) g^{\prime}\left(x_{1}\right) v=k^{\prime}\left(x_{1}\right) f^{\prime}\left(x_{1}\right)^{-1} v \\
& =k^{\prime}\left(x_{1}\right) f^{\prime}\left(x_{1}\right)^{-1} \frac{f\left(x_{1}\right)-f\left(x_{0}\right)}{t_{0}} \quad \text { for } t_{0}>0 .
\end{aligned}
$$

Comparison with formula (1.2) shows that this derivative is nonnegative, and the function $t \mapsto k\left(g\left(y_{0}+t v\right)\right)$ is indeed locally weakly increasing for $t \geq 0$.

Conversely, suppose that $t \mapsto k\left(g\left(y_{0}+t v\right)\right)$ is weakly increasing for $t \geq 0$ for any choice of $v$ and $g$. Let $x \in \Omega \backslash\left\{x_{0}\right\}, U$ be a neighbourhood of $x_{0}$ where $f$ is one-to-one and let $g:=\left(\left.f\right|_{U}\right)^{-1}$. Let also $v:=f(x)-y_{0}$. Since the function $t \mapsto k\left(g\left(y_{0}+t v\right)\right)$ is defined and weakly increasing at $t=1$, the derivative must be nonnegative, that is to say, 


$$
\begin{aligned}
0 & \leq k^{\prime}\left(g\left(y_{0}+v\right)\right) f^{\prime}\left(g\left(y_{0}+v\right)\right)^{-1} \frac{f\left(g\left(y_{0}+v\right)\right)-f\left(x_{0}\right)}{1} \\
& =k^{\prime}(x) f^{\prime}(x)^{-1}\left(f(x)-f\left(x_{0}\right)\right)
\end{aligned}
$$

which is precisely equation (1.2).

Proof of Proposition 1.3. It is obvious that the function $k(x):=$ $1 /\left(r_{0}-\left|x-x_{0}\right|\right)$ is continuous, proper and bounded from below on $B_{r_{0}}\left(x_{0}\right)$. It is also differentiable outside $x_{0}$ and its differential $k^{\prime}(x)$ applied to the vector $v \in \mathbb{R}^{n}$ is

$$
k^{\prime}(x) v=\frac{1}{\left(r_{0}-\left|x-x_{0}\right|\right)^{2}} \frac{x-x_{0}}{\left|x-x_{0}\right|} \cdot v .
$$

To conclude we only have to substitute $f^{\prime}(x)^{-1}\left(f(x)-f\left(x_{0}\right)\right)$ for $v$ and apply Proposition 1.2 and Theorem 1.1.

EXAMPLE 4.1. A bilipschitz homeomorphism of the plane $\mathbb{R}^{2}$ onto itself that leaves the origin fixed but maps no ball centred at the origin onto a star-shaped set is not hard to describe. (In the following arguments, "starshapedness" will be always understood with respect to the origin.) The balls will first be elongated by a linear transformation $g$ :

$$
g\left(x_{1}, x_{2}\right):=\left(x_{1}, c x_{2}\right) \quad \text { for }\left(x_{1}, x_{2}\right) \in \mathbb{R}^{2}, \text { with } c=e^{\pi / 2},
$$

and then twisted around by a second mapping $h$ into a non-star-shaped set. This $h$ is best written in polar coordinates:

$$
(\varrho, \theta) \mapsto(\varrho, \theta-\ln \varrho) .
$$

Figure 1 shows the effect of the composition $f:=h \circ g$, which is a bilipschitz homeomorphism of the plane onto itself, on a few of the balls $B_{r}$. Indeed, these images appear not to be star-shaped and it is not difficult to prove it analytically.

It is interesting to note that the mappings $g, h, f$ that we have described are special cases of the homeomorphisms that are used to classify the flows of linear ordinary differential equations up to topological equivalence. For example, $h$ transforms the solutions of the two-dimensional diagonal system

$$
\left(\begin{array}{l}
\dot{x}_{1} \\
\dot{x}_{2}
\end{array}\right)=\left(\begin{array}{ll}
1 & 0 \\
0 & 1
\end{array}\right)\left(\begin{array}{l}
x_{1} \\
x_{2}
\end{array}\right)
$$

into the solutions of

$$
\left(\begin{array}{l}
\dot{x}_{1} \\
\dot{x}_{2}
\end{array}\right)=\left(\begin{array}{cc}
1 & 1 \\
-1 & 1
\end{array}\right)\left(\begin{array}{l}
x_{1} \\
x_{2}
\end{array}\right) .
$$

See, e.g., [2], Ch. 3, $\S 21-22$.

The following three propositions are variants of invertibility criteria found in [26], [10] and [27]. The original results are not contained in the ones below because more smoothness is asked of the function $k$ here, but this 


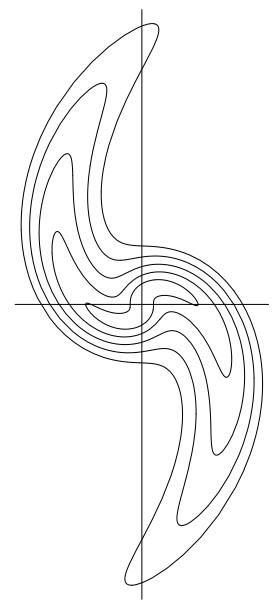

Fig. 1

is only to avoid some uninteresting formal complications in the statement and in the proof. Moreover, everything here is finite-dimensional. However, Propositions 4.3 and 4.4 do generalize the old results in that the $\omega$ 's, that here are functions, used to be constants in [27].

Proposition 4.2. Let $\Omega$ be a nonempty, open and connected subset of $\mathbb{R}^{n}, f: \Omega \rightarrow \mathbb{R}^{n}$ be a local $C^{1}$ diffeomorphism and $k: \Omega \rightarrow \mathbb{R}$ be a $C^{1}$ function. Suppose that the set $C:=\{x \in \Omega \mid k(x) \leq c\}$, for a given constant $c \in \mathbb{R}$, is compact and connected and that there exists $x_{0} \in C$ such that

$$
k^{\prime}(x) f^{\prime}(x)^{-1}\left(f(x)-f\left(x_{0}\right)\right)>0 \quad \text { for all } x \in \Omega \text { such that } k(x)=c .
$$

Then $f$ is one-to-one on $C$ and $f(C)$ is star-shaped with respect to $f\left(x_{0}\right)$.

Proof. Inequality (4.8) implies in particular that $k^{\prime}(x) \neq 0$ for all $x$ such that $k(x)=c$ and that $k\left(x_{0}\right) \neq c$. Then the set $\{x \in \Omega \mid k(x)<c\}$ contains $x_{0}$ and its boundary is the $C^{1}$ manifold $\{x \in \Omega \mid k(x)=c\}$.

We are going to verify that condition (b) of Proposition 2.7 holds. Let $v \in \mathbb{R}$ be an arbitrary vector and consider the path $\gamma_{x_{0}, v}$. Proposition 2.1 assures us that the path will eventually leave the compact set $C$. Let $\bar{t}>0$ be the instant when the trajectory first hits the boundary $\partial C$, at $\bar{x}=\gamma_{x_{0}, v}(\bar{t})$. The derivative of $k$ along $\gamma_{x_{0}, v}$ at $\bar{t}$ is positive by assumption (4.8):

$$
\left.\frac{d}{d t} k\left(\gamma_{x_{0}, v}(t)\right)\right|_{t=\bar{t}}=k^{\prime}(\bar{x}) f^{\prime}(\bar{x})^{-1}\left(f(\bar{x})-f\left(x_{0}\right)\right)>0 .
$$

Hence $k\left(\gamma_{x_{0}, v}(t)\right)>c$ for any time $t$ slightly later than $\bar{t}$. Actually, at no later time can the value of $k$ return to $c$, because at the earliest time $\widetilde{t}$ the path happened to hit again the boundary $\partial C$, the value $c$ ought to be approached from above, which is incompatible with the derivative condition (4.8). This 
shows that $\gamma_{x_{0}, v}^{-1}(C) \cap[0, \infty[$ is a (compact) interval containing 0 . The reasoning is perfectly symmetrical for the negative times.

Proposition 4.3. Let $\Omega$ be a nonempty, open and connected subset of $\mathbb{R}^{n}, f: \Omega \rightarrow \mathbb{R}^{n}$ be a local $C^{1}$ diffeomorphism. Suppose that there exist a proper, bounded below $C^{1}$ function $k: \Omega \rightarrow \mathbb{R}$, a locally integrable function $\omega:\left[0, \infty\left[\rightarrow\left[0, \infty\left[\right.\right.\right.\right.$ and a point $x_{0} \in \Omega$ such that

$$
k^{\prime}(x) f^{\prime}(x)^{-1}\left(f(x)-f\left(x_{0}\right)\right) \geq-\omega\left(\left|f(x)-f\left(x_{0}\right)\right|\right) \quad \text { for all } x \in \Omega .
$$

Then $f$ is one-to-one on $\Omega$ and $f(\Omega)$ is star-shaped with respect to $f\left(x_{0}\right)$.

Proof. Let us verify that condition (b)' of Remark 2.12 holds for the following function:

$$
\begin{aligned}
& \widetilde{k}(x):=k(x)+\int_{1}^{\left|f(x)-y_{0}\right|} \frac{\omega(s)}{s} d s \\
& \text { for the } x \in \Omega \text { such that } f(x) \neq y_{0},
\end{aligned}
$$

where $y_{0}:=f\left(x_{0}\right)$. It is easily checked that $\widetilde{k}$ is continuous. The set

$$
\left\{x \in \Omega|\widetilde{k}(x) \leq a,| f(x)-y_{0} \mid \geq \varepsilon\right\}
$$

is compact because it is a closed subset of the compact set

$$
\left\{x \in \Omega \mid \inf k \leq k(x) \leq a-\int_{1}^{\varepsilon} \frac{\omega(s)}{s} d s\right\} .
$$

Let $g$ be a local inverse of $f$ whose domain intersects the half-line $\left\{y_{0}+t v \mid\right.$ $t>0\}$ for a given vector $v \in \mathbb{R}^{n} \backslash\{0\}$. Then the function

$$
t \mapsto \widetilde{k}\left(g\left(y_{0}+t v\right)\right)
$$

is absolutely continuous and its derivative is

$$
\begin{aligned}
k^{\prime}\left(g\left(y_{0}+t v\right)\right) f^{\prime}\left(g\left(y_{0}+t v\right)\right)^{-1} v+\frac{\omega(t|v|)}{t|v|}|v| \\
=k^{\prime}\left(g\left(y_{0}+t v\right)\right) f^{\prime}\left(g\left(y_{0}+t v\right)\right)^{-1}\left(f\left(g\left(y_{0}+t v\right)\right)-y_{0}\right) \cdot \frac{1}{t} \\
\quad+\omega\left(\left|f\left(g\left(y_{0}+t v\right)\right)-y_{0}\right|\right) \cdot \frac{1}{t} \geq 0
\end{aligned}
$$

for almost every $t>0$ where it is defined. Hence it is pointwise weakly increasing on its (open) domain and we are done.

Proposition 4.4. Let $\Omega$ be a nonempty, open and connected subset of $\mathbb{R}^{n}$, and $f: \Omega \rightarrow \mathbb{R}^{n}$ be a local $C^{1}$ diffeomorphism. Then the following two conditions are equivalent: 
(a) $f$ is one-to-one and $f(\Omega)=\mathbb{R}^{n}$;

(b) there exist a proper $C^{1}$ function $k: \Omega \rightarrow \mathbb{R}$ bounded from below, a locally integrable function $\omega:\left[0, \infty\left[\rightarrow\left[0, \infty\left[\right.\right.\right.\right.$ and a point $x_{0} \in \Omega$ such that

$$
k^{\prime}(x) f^{\prime}(x)^{-1}\left(f(x)-f\left(x_{0}\right)\right) \leq \omega\left(\left|f(x)-f\left(x_{0}\right)\right|\right) \quad \text { for all } x \in \Omega .
$$

Proof. If (a) holds, then we can set $x_{0} \in \Omega$ arbitrary, $k(x):=\mid f(x)-$ $\left.f\left(x_{0}\right)\right|^{2}, \omega(r):=2 r^{2}$. For the converse, we are going to build a function $\varphi$ so that Proposition 2.9 can be applied. As usual, $y_{0}$ will denote $f\left(x_{0}\right)$.

Let $\varepsilon>0$ be the radius of a closed ball centred at $x_{0}$ and contained in $\mathcal{A}$ and let $\delta>0$ be such that $B_{\delta}\left(y_{0}\right) \subset f\left(B_{\varepsilon}\left(x_{0}\right)\right)$. In particular, $f$ is one-to-one on $B_{\varepsilon}\left(x_{0}\right)$. Define

$$
\varphi_{0}(r):=\sup \left\{k(x)|| x-x_{0}|\leq \varepsilon,| f(x)-y_{0} \mid \leq r\right\} \quad \text { for } r \geq 0 .
$$

It is obvious that this $\varphi_{0}$ is continuous and weakly increasing and that

$$
k(x) \leq \varphi_{0}\left(\left|f(x)-y_{0}\right|\right) \quad \text { for } x \in B_{\varepsilon}\left(x_{0}\right) .
$$

Let now $x \in \mathcal{A} \backslash B_{\varepsilon}\left(x_{0}\right)$ and let $\gamma:=\gamma_{x_{0}, f(x)-y_{0}}$. We have

$$
\begin{aligned}
\gamma(t) \in B_{\varepsilon}\left(x_{0}\right), \quad f(\gamma(t))= & y_{0}+t\left(f(x)-y_{0}\right) \in B_{\delta}\left(y_{0}\right) \\
& \text { whenever }|t| \leq \frac{\delta}{\left|f(x)-y_{0}\right|}=: t_{0} .
\end{aligned}
$$

We can write

$$
\begin{aligned}
k(x) & =k(\gamma(1))=k\left(\gamma\left(t_{0}\right)\right)+\left(k(\gamma(1))-k\left(\gamma\left(t_{0}\right)\right)\right) \\
& =k\left(\gamma\left(t_{0}\right)\right)+\int_{t_{0}}^{1} \frac{d}{d t} k(\gamma(t)) d t \\
& =k\left(\gamma\left(t_{0}\right)\right)+\int_{t_{0}}^{1} k^{\prime}(\gamma(t)) f^{\prime}(\gamma(t))^{-1}\left(f(x)-y_{0}\right) d t \\
& =k\left(\gamma\left(t_{0}\right)\right)+\int_{t_{0}}^{1} k^{\prime}(\gamma(t)) f^{\prime}(\gamma(t))^{-1}\left(f(\gamma(t))-y_{0}\right) \frac{1}{t} d t \\
& \leq \varphi_{0}(\delta)+\int_{t_{0}}^{1} \omega\left(\left|f(\gamma(t))-y_{0}\right|\right) \frac{1}{t} d t \\
& =\varphi_{0}(\delta)+\int_{\delta /\left|f(x)-y_{0}\right|}^{1} \omega\left(t\left|f(x)-y_{0}\right|\right) \frac{1}{t} d t \\
& =\varphi_{0}(\delta)+\int_{\delta}^{\left|f(x)-y_{0}\right|} \frac{\omega(s)}{s} d s .
\end{aligned}
$$


If we define

$$
\varphi(r):=\varphi_{0}(r)+\int_{\delta}^{\max \{r, \delta\}} \frac{\omega(s)}{s} d s \quad \text { for } r \geq 0,
$$

we easily see that the resulting $\varphi$ is locally bounded (actually, it is continuous and weakly increasing) and that

$$
k(x) \leq \varphi\left(\left|f(x)-y_{0}\right|\right) \quad \text { for all } x \in \mathcal{A} .
$$

As announced, the conclusion follows from Proposition 2.9. Note that we did not use formula (4.16) at $x=x_{0}$, so that we actually did not need to assume $k$ to be differentiable at $x_{0}$.

Corollary 4.5. Let $f: \mathbb{R}^{n} \rightarrow \mathbb{R}^{n}$ be a local $C^{1}$ diffeomorphism. Suppose that there exist a point $x_{0} \in \mathbb{R}^{n}$ and two functions $\alpha, \omega:[0, \infty[\rightarrow[0, \infty[$, of which $\omega$ is locally integrable and $\alpha$ is also continuous, strictly positive and satisfying the integral condition

$$
\int_{0}^{\infty} \frac{r}{\alpha(r)} d r=\infty
$$

in such a way that

$$
\begin{aligned}
& \left(x-x_{0}\right) \cdot f^{\prime}(x)^{-1}\left(f(x)-f\left(x_{0}\right)\right) \\
& \quad \leq \alpha\left(\left|x-x_{0}\right|\right) \omega\left(\left|f(x)-f\left(x_{0}\right)\right|\right) \quad \text { for all } x \in \mathbb{R}^{n} .
\end{aligned}
$$

Then $f$ is one-to-one and $f\left(\mathbb{R}^{n}\right)=\mathbb{R}^{n}$.

Proof. Define

$$
k(x):=\int_{0}^{\left|x-x_{0}\right|} \frac{r}{\alpha(r)} d r \quad \text { for } x \in \mathbb{R}^{n} .
$$

This function $k$ is $C^{1}$ on all of $\mathbb{R}^{n}$ (even at $x_{0}$, although we may find the way not to use this fact); it is obviously bounded from below and it is also proper as a function from $\mathbb{R}^{n}$ to $\mathbb{R}$ because of assumption (4.23). Then we can apply Proposition 4.4:

$$
\begin{aligned}
k^{\prime}(x) f^{\prime}(x)^{-1}(f(x)- & \left.f\left(x_{0}\right)\right) \\
& =\frac{\left|x-x_{0}\right|}{\alpha\left(\left|x-x_{0}\right|\right)} \frac{x-x_{0}}{\left|x-x_{0}\right|} \cdot f(x)^{-1}\left(f(x)-f\left(x_{0}\right)\right) \\
& \leq \omega\left(\left|f(x)-f\left(x_{0}\right)\right|\right) \quad \text { for all } x \in \mathbb{R}^{n} \backslash\left\{x_{0}\right\}
\end{aligned}
$$

(the inequality also holds for $x=x_{0}$ ).

Corollary 4.6 (Hadamard). Let $f: \mathbb{R}^{n} \rightarrow \mathbb{R}^{n}$ be a $C^{1}$ local diffeomorphism. Suppose that there exists a continuous function $\beta:[0, \infty[\rightarrow] 0, \infty[$ satisfying the integral condition 


$$
\int_{0}^{\infty} \frac{1}{\beta(r)} d r=\infty
$$

and such that

$$
\left\|f^{\prime}(x)^{-1}\right\| \leq \beta(|x|) \quad \text { for all } x \in \mathbb{R}^{n} .
$$

Then $f$ is one-to-one and $f\left(\mathbb{R}^{n}\right)=\mathbb{R}^{n}$.

P r o o f. Apply Corollary 4.5 above with $x_{0}:=0, \alpha(r):=(1+r) \beta(r)$ and $\omega(r):=r$.

It is easy to verify that, if the last corollary above can be applied to a mapping $f$, then the function

$$
k(x):=\int_{0}^{|x|} \frac{1}{\beta(r)} d r+\left|f(x)-f\left(x_{0}\right)\right| \quad \text { for } x \in \mathbb{R}^{n}
$$

is nonnegative, continuous and proper as a mapping from $\mathbb{R}^{n}$ to $\mathbb{R}$ and satisfies

$$
k^{\prime}(x) f^{\prime}(x)^{-1}\left(f(x)-f\left(x_{0}\right)\right) \geq 0 \quad \text { for } x \neq x_{0},
$$

so that it is an auxiliary function for $f$ in the sense of Theorem 1.1. In particular, for example, $f$ maps every set of the form $\left\{x \in \mathbb{R}^{n} \mid k(x) \leq c\right\}$ onto a set which is star-shaped with respect to $f\left(x_{0}\right)$.

Acknowledgements. We are grateful to Professor Czesław Olech for some stimulating comments on a previous version of the paper.

\section{References}

[1] A. Ambrosetti and G. Prodi, On the inversion of some differentiable mappings with singularities between Banach spaces, Ann. Mat. Pura Appl. 93 (1973), 231-247.

[2] V. I. Arnol'd, Ordinary Differential Equations, 3rd ed., Springer, 1992.

[3] S. Banach and S. Mazur, Über mehrdeutige stetige Abbildungen, Studia Math. 5 (1934), 174-178.

[4] N. P. Bhatia and G. P. Szegö, Stability Theory of Dynamical Systems, Springer, 1970.

[5] F. Browder, Covering spaces, fiber spaces and local homeomorphisms, Duke Math. J. 21 (1954), 329-336.

[6] R. Cacciop poli, Sugli elementi uniti delle trasformazioni funzionali: un teorema di esistenza e di unicità ed alcune sue applicazioni, Rend. Sem. Mat. Univ. Padova 3 (1932), 1-15.

[7] L. M. Drużkowski, The Jacobian Conjecture, Preprint 429, Institute of Mathematics, Jagiellonian University, Kraków, 1990.

[8] P. L. Duren, Univalent Functions, Springer, 1983.

[9] D. Gale and H. Nikaido, The Jacobian matrix and global univalence of mappings, Math. Ann. 159 (1965), 81-93. 
[10] G. Gorni, A criterion of invertibility in the large for local diffeomorphisms between Banach spaces, Nonlinear Anal. 21 (1993), 43-47.

[11] J. Hadamard, Sur les transformations ponctuelles, Bull. Soc. Math. France 34 (1906), 71-84.

[12] P. Lévy, Sur les fonctions de lignes implicites, ibid. 48 (1920), 13-27.

[13] G. H. Meisters, Inverting polynomial maps of n-space by solving differential equations, in: Fink, Miller, Kliemann (eds.), Delay and Differential Equations, Proceedings in Honour of George Seifert on his retirement, World Sci., 1992, 107-166.

[14] G. H. Meisters and C. Olech, Locally one-to-one mappings and a classical theorem on schlicht functions, Duke Math. J. 30 (1963), 63-80.

[15] - - - Solution of the global asymptotic stability Jacobian conjecture for the polynomial case, in: Analyse Mathématique et Applications, Gauthier-Villars, Paris, 1988, 373-381.

[16] C. Olech, Global diffeomorphism questions and differential equations, in: Qualitative Theory of Differential Equations, Szeged, 1988, Colloq. Math. Soc. János Bolyai 53, North-Holland, 1990, 465-471.

[17] J. M. Ortega and W. C. Rheinboldt, Iterative Solutions of Nonlinear Equations in Several Variables, Academic Press, 1970.

[18] T. Parthasarathy, On Global Univalence Theorems, Lecture Notes in Math. 977, Springer, 1983.

[19] R. Plastock, Homeomorphisms between Banach spaces, Trans. Amer. Math. Soc. 200 (1974), 169-183.

[20] P. J. Rabier, On global diffeomorphisms of Euclidian space, Nonlinear Anal. 21 (1993), 925-947.

[21] M. Rădulescu and S. Rădulescu, Global inversion theorems and applications to differential equations, ibid. 4 (1980), 951-965.

[22] W. C. Rheinboldt, Local mapping relations and global implicit function theorems, Trans. Amer. Math. Soc. 138 (1969), 183-198.

[23] J. Sotomayor, Inversion of smooth mappings, Z. Angew. Math. Phys. 41 (1990), 306-310.

[24] M. Spivak, A Comprehensive Introduction to Differential Geometry, Publish or Perish, Houston, Tex., 1970, 1979.

[25] T. Ważewski, Sur l'évaluation du domaine d'existence de fonctions implicites réelles ou complexes, Ann. Soc. Polon. Math. 20 (1947), 81-120.

[26] G. Zampieri, Finding domains of invertibility for smooth functions by means of attraction basins, J. Differential Equations 104 (1993), 11-19.

[27] —, Diffeomorphisms with Banach space domains, Nonlinear Anal. 19 (1992), 923932.

[28] G. Zampieri and G. Gorni, On the Jacobian conjecture for global asymptotic stability, J. Dynamics Differential Equations 4 (1992), 43-55.

DIPARTIMENTO DI MATEMATICA E INFORMATICA

UNIVERSITÀ DI UDINE

VIA ZANON 6

33100 UDINE, ITALY

E-mail: GORNI@UDMI5400.CINECA.IT

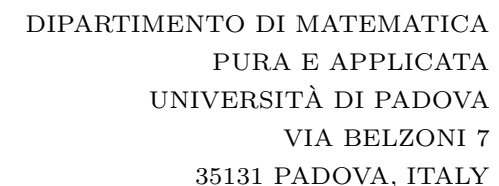

E-mail: GAETANO@PDMAT1.UNIPD.IT 